\title{
Feature Complementarity and Assortment in Choice
}

\author{
ALEXANDER CHERNEV*
}

\begin{abstract}
This research argues that the purchase probability from a given choice set is contingent on the complementarity of the features differentiating its options. In particular, two types of features are distinguished: complementary features, which are characterized by the additivity of their utilities, and noncomplementary features, which are characterized by nonadditive utilities. In this context, it is argued that assortments in which options are differentiated by noncomplementary features are likely to be associated with a greater probability of purchase than assortments with options differentiated by complementary features. This prediction is supported by data from three experimental studies. The article concludes with a discussion of the theoretical implications and offers directions for further research.
\end{abstract}

$\mathrm{C}$ onsider a consumer who is choosing toothpaste among offerings by two different brands. The first brand offers four toothpastes differentiated by flavor: lemon, cinnamon, banana, and mint. The second brand offers four toothpastes differentiated by functional attributes: cavity prevention, tartar protection, teeth whitening, and breath freshening. Which of the two brands is more likely to be chosen by the consumer?

From a conceptual standpoint, the two brands in the above example differ in terms of the degree to which features that differentiate options in their product lines complement one another. Thus, combining two or more features differentiating products offered by the first brand does not necessarily increase their overall utility. For example, blending cinnamon and mint flavors does not create a superior combination. In contrast, features differentiating products offered by the second brand do complement one another, and their mix is associated with a greater utility than any of the individual features considered separately. For example, combining cavity prevention and tartar protection leads to a superior combination.

Extant marketing and decision literature does not explicitly address the issue of how feature complementarity influences choice. In fact, prior research does not differentiate between the two scenarios described in the above example, and, as a result, it is implicitly assumed that complementary and noncomplementary features will have a similar impact on choice. In contrast, the research presented in this article argues that feature complementarity plays an important role in consumers' product evaluations and choice. In particular,

*Alexander Chernev is associate professor of marketing, Kellogg School of Management, Northwestern University, 2001 Sheridan Road, Evanston, IL 60208 (ach@northwestern.edu). The author thanks the editor, the associate editor, and the three reviewers for their advice and constructive comments. it is proposed that choice sets in which options are differentiated by complementary features are associated with a lower choice probability than sets in which options are differentiated by noncomplementary features. This proposition is articulated in a set of research hypotheses and empirically tested in a series of three experiments.

\section{THEORETICAL BACKGROUND}

\section{Feature Complementarity in Choice}

In microeconomics, goods are considered complements if an increase (decrease) in the price of one good leads to a decrease (increase) in the quantity demanded of the other. A typical example of complementary goods is gasoline and motor oil: if gasoline consumption falls because of a price increase, the consumption of motor oil will fall as well, since gasoline and motor oil are used together (Pindyck and Rubinfeld 2001). In this context, complements are often contrasted with substitutes because for substitutes the pricequantity relationship is reversed: an increase (decrease) in the price of one good leads to an increase (decrease) in the quantity demanded of the other (e.g., movie tickets and video rentals).

In marketing, complementarity is often defined relative to product-specific utilities and the corresponding consumer needs, rather than through products' cross-price elasticities. In this context, complementary products are defined as those chosen to fill different aspects of a consumer's composite need (and hence usually consumed jointly), whereas substitute products are defined as those chosen to fill the same aspect of a consumer's need (Lattin and McAlister 1985 and Walters 1991; see also Henderson and Quandt 1958). In this context, product complementarity has been measured by the degree to which products tend to be consumed jointly, 
so that the consumption of one product enhances the consumption of the other.

This article extends the notion of complementarity beyond overall product descriptions to particular features that describe choice alternatives. In this context, complementarity is used in reference to the additivity of feature-specific utilities and, in particular, in reference to the marginal utility that one feature adds in the presence of the other. Thus, the addition of a complementary feature (e.g., tartar protection) to a product that already has a similar feature (e.g., cavity prevention) tends to make the overall product more attractive. In contrast, adding a noncomplementary feature (e.g., mint flavor) to a product that already has a similar feature (e.g., banana flavor) does not increase the overall product attractiveness.

Feature complementarity can be more formally presented as follows. If $U_{A}$ is the utility associated with feature $A, U_{B}$ is the utility associated with feature $\mathrm{B}$, and $\mathrm{U}_{\mathrm{AB}}$ is the utility associated with features $\mathrm{A}$ and $\mathrm{B}$ combined, then $\mathrm{A}$ and $\mathrm{B}$ are complementary when $\mathrm{U}_{\mathrm{AB}}>\mathrm{U}_{\mathrm{A}}$ and $\mathrm{U}_{\mathrm{AB}}>\mathrm{U}_{\mathrm{B}}$. In contrast, $\mathrm{A}$ and $\mathrm{B}$ are noncomplementary when $\mathrm{U}_{\mathrm{AB}} \leq \mathrm{U}_{\mathrm{A}}$ and $\mathrm{U}_{\mathrm{AB}} \leq \mathrm{U}_{\mathrm{B}}$. Note, however, that, in reality, feature complementarity is better represented as a continuum such that different features vary in their degree of complementarity; the binary classification of features into complementary and noncomplementary is done primarily for parsimony of the theoretical development and analysis.

On a conceptual level, feature complementarity can be related to the notion of alignability, which has been advanced by research on structural alignment in similarity comparisons (Gentner 1983; Gentner and Markman 1994). It has been proposed that the structural alignment process yields two types of relationships: commonalities and differences, which, in turn, can be classified as alignable or nonalignable (Markman and Gentner 1993). Both alignable and nonalignable differences describe the relationship between the attribute levels of the compared objects; however, nonalignable differences describe a scenario in which a given feature is present in one of the objects and absent in the other, whereas alignable differences describe a scenario in which objects have different (but nonzero) levels of a given attribute. A typical example compares cameras and camcorders; the fact that cameras take still pictures and camcorders take moving pictures is viewed as an alignable difference, whereas the fact that camcorders record sound and cameras do not is a nonalignable difference (Markman and Medin 1995).

Although conceptually similar, complementarity and alignability differ in two key aspects. First, alignability implies a similarity-based comparison process in which individuals simply match the features between the two objects. The goal of this process, therefore, is to establish the degree of similarity between the objects; the utilities associated with these objects and their attributes are irrelevant. In contrast, complementarity implies a choice process, in which individuals evaluate alternatives and their features with respect to their utility. Feature matching, although possible, is not a prerequisite for judging complementarity and choice.

The differences in the processes underlying alignability and complementarity judgments are further reflected in the fact that both complementary and noncomplementary features can be classified as alignable by the structural alignment theory. Thus, noncomplementary features (e.g., different flavors) are considered alignable because all alternatives have corresponding features. Similarly, as long as all options have nonzero levels of performance on the attributes defined by the complementary features, these features would be considered alignable as well (Markman and Gentner 1993). To illustrate, option A's superiority on a given attribute (e.g., cavity prevention) does not imply that option B entirely lacks that attribute, but simply that option $\mathrm{B}$ underperforms on that attribute relative to option A.

\section{Hypothesis Development}

A central tenet of this research is that feature complementarity can have a significant impact on choice. In particular, it is proposed that choice sets differentiated by complementary features are likely to be associated with a lower likelihood of purchase than choices from sets differentiated by noncomplementary features. This proposition builds on the notion that because complementary features are relatively independent from one another, they are perceived as levels of separate attribute dimensions rather than as levels of the same attribute. As a result, in the case of complementary features, consumers are likely to opt for the best performance on each attribute, and the absence of a given feature is then viewed as a loss. This leads to the counterintuitive prediction that differentiating options by complementary features can actually decrease the attractiveness of each individual option.

Consider an offering featuring a single attribute (e.g., cavity prevention toothpaste). Adding an option differentiated by a complementary feature (e.g., tartar control) highlights an attribute dimension on which the original product is inferior, thus decreasing its overall attractiveness. Furthermore, it can be argued that each new complementary feature used to extend the product line ultimately makes the extant products less attractive because they are dominated on the attribute made salient by the newly added feature. In this context, it is proposed that increasing product assortment by adding options differentiated by complementary features could potentially lower the attractiveness of all alternatives in that assortment.

The above discussion is illustrated in figure 1. Panel A depicts a binary attribute scenario in which product $\mathrm{A}$ is extended by adding two complementary features. Because the utility of each of feature is relatively independent of the other, these features are represented as two orthogonal attribute dimensions. In this context, $\mathrm{A}, \mathrm{A}^{\prime}$, and $\mathrm{A}^{\prime \prime}$ comprise the extended set in which $\mathrm{A}^{\prime}$ dominates $\mathrm{A}$ on dimension 1 and $\mathrm{A}^{\prime \prime}$ dominates $\mathrm{A}$ on dimension 2. Because the scales underlying dimensions 1 and 2 are ordinal (higher quantities of a given attribute are preferred to lower quantities), options 
FIGURE 1

PRODUCT DIFFERENTIATION AND FEATURE COMPLEMENTARITY IN CHOICE

A. Product line extensions differentiated by complementary features

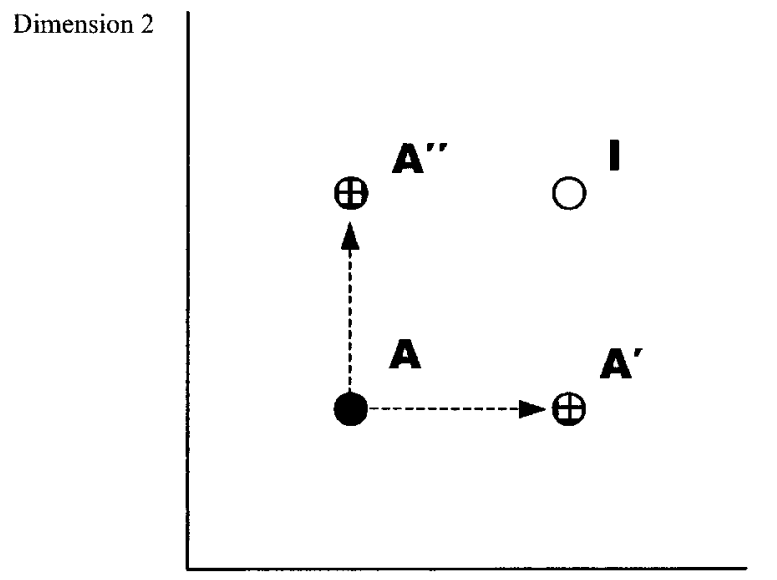

Dimension 1

B. Product line extensions differentiated by noncomplementary features

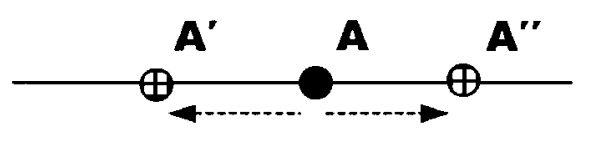

Dimension 1

$\mathrm{A}^{\prime}$ and $\mathrm{A}^{\prime \prime}$ define the ideal point $\mathrm{I}$, which combines both features. To illustrate, when choosing among alternatives featuring cavity prevention and tartar protection, consumers' ideal toothpaste is defined by the presence of both features. In this context, all three options are likely to be viewed as suboptimal relative to the ideal because both $\mathrm{A}^{\prime}$ and $\mathrm{A}^{\prime \prime}$ have disadvantages relative to one another, and the original product $\mathrm{A}$ is dominated by both $\mathrm{A}^{\prime}$ and $\mathrm{A}^{\prime \prime}$. ${ }^{1}$

Unlike complementary features, the noncomplementary features tend to be mutually exclusive, and, as a result, they are likely to be treated as different levels of the same attribute (fig. 1, panel B). Furthermore, unlike the case with complementary features, the scale underlying dimension 1 is nominal, and consumers' ideal point depends to a large degree on their individual preferences. Given that the benefits offered by these features are nonadditive, combining two or more features does not bring their combination closer to a consumer's ideal point.

Therefore, this research posits that the complementarity of the features differentiating options in a given choice set

\footnotetext{
${ }^{1}$ This discussion naturally raises the question of whether the potential adverse effect of complementary features can be alleviated by introducing the ideal option. Such an approach, however, defeats the very purpose of introducing options $\mathrm{A}^{\prime}$ and $\mathrm{A}^{\prime \prime}$ since they will be clearly inferior to the ideal option I.
}

has a significant impact on the overall probability of purchase and choice deferral. Because differentiation by complementary features highlights the deficiencies of the other options, it is proposed that consumers will be less likely to choose from sets in which options are differentiated by complementary features than from sets in which options are differentiated by noncomplementary features. This prediction can be more formally stated as follows:

H1: The complementarity of features differentiating choice alternatives influences the overall purchase probability from the set. In particular, choice deferral will be greater for sets in which options are differentiated by complementary than by noncomplementary features.

This hypothesis is tested in the following experiment.

\section{EXPERIMENT 1}

\section{Method}

Seventy-three Northwestern University undergraduates were respondents. They were informed that the choice task involved making hypothetical purchase decisions in several product categories. Respondents were also told that there were no right or wrong answers and that they should consider only their own preferences.

Two product categories were used as stimuli: an MP3 player and a Caribbean resort. Similar categories have been successfully used in prior research (Chernev 2003b; Shafir 1993). Choice sets consisted of five options, each described on four attributes. Feature complementarity was manipulated by varying the nature of the attribute differentiating choice alternatives. Each option was described by four attributesone attribute composed of either complementary or noncomplementary features and three attributes composed of common features. Thus, in the complementary condition, the MP3 players varied in terms of options (user-friendly design, extended battery life, clear sound, extra sturdy construction, and shock-resistant play) and had three common features: memory, weight, and warranty. In the noncomplementary condition, the MP3 players had the same three common features but varied in color (silver, white, black, blue, or red) rather than in terms of options. In the Caribbean resort scenario, alternatives in the complementary condition were featured as follows: fantastic beaches, convenient transportation, vibrant nightlife, excellent restaurants, and exceptional service. In the noncomplementary condition, options were differentiated based on their location (Bermuda, Bahamas, Barbados, Antigua, and Aruba). The common features in both conditions were the resort category, number of swimming pools, and room service. To avoid potential pricequality inferences, options were said to be equally priced. A summary of the experimental stimuli is given in appendix table A1.

Respondents were first asked to rate the attractiveness of each of the complementary and noncomplementary features, using an 11-point scale $(0=$ not attractive at all; $10=$ 
very attractive). To illustrate, in the case of an MP3 player, respondents were asked to rate the attractiveness of the five complementary features and were then asked to evaluate the attractiveness of the five noncomplementary features. Next, respondents were asked to rate the relative attractiveness of those features by allocating 10 points across each of the sets of five features. To illustrate, respondents who considered different features to be equally attractive had to allocate two points for each of these features.

Next, respondents were given an unrelated filler task followed by a choice task in which they were asked to evaluate four sets of five alternatives. Respondents were asked to choose one of the options and were then given the option to either stay with their original selection or to postpone their choice and look for other options. At the end of the experiment respondents were debriefed and paid $\$ 5$ for participating.

\section{Results}

Manipulation Check. A key assumption of the experimental procedure was that the product features given in appendix table A1 varied in their complementarity. The validity of this assumption was pretested using a different sample of 23 respondents from the same population. The experimental task asked respondents to create their "ideal" product by using some or all of the available attributes. For each product category there were two decisions: one for features presumed to be complementary and one for features presumed to be noncomplementary. Each respondent made two ideal-product decisions-one per product category. To avoid potential product category and feature complementarity confounds, the design was counterbalanced: 12 of the respondents were given a combination in which the MP3 player was described on complementary features (while the resort was described on noncomplementary features). The remaining 11 respondents were given a combination in which the MP3 player was described on noncomplementary features.

For each product decision, respondents were given a list of features and asked to use those features to create their ideal product. To illustrate, for one of the choices respondents were given the features of user-friendly design, extended battery life, clear sound, extra sturdiness, shockresistant play, 2,500-song memory, light weight, and 12 mo. warranty. Respondents were then instructed that they could select any number of features (from a single feature to all features) that best described their ideal MP3 player.

The data show that the total number of features used to describe the respondents' ideal point varied across the two feature types. The mean for the complementary features was significantly higher than the mean for the noncomplementary features $(M=4.56$ vs. $M=1.69 ; F(1,22)=97.8$, $p<.001)$. This finding is consistent with the proposition that a combination of complementary features is likely to yield a greater utility than a combination of noncomplementary features.
Another important assumption was that the complementary features were not perceived to be less attractive than the noncomplementary features. Thus, the greater probability of purchase from noncomplementary assortments, predicted in hypothesis 1 , could not be attributed to the higher overall attractiveness of the noncomplementary assortments. The validity of this assumption was examined by comparing the respondents' ratings of the attractiveness of the complementary versus noncomplementary features. Thus, for both complementary and noncomplementary features, summary attractiveness ratings were calculated that averaged respondents' ratings of the attractiveness of each of the five features. The data show that, on average, complementary features were perceived to be more attractive than noncomplementary features $(M=7.92$ vs. $M=6.78 ; F(1,216)=$ $78.3, p<.001)$. This effect varied in strength across the two product categories: it was more pronounced for the MP3 player $(M=7.95$ vs. $M=6.10)$ than for the Caribbean resort $(M=7.90$ vs. $M=7.46 ; F(1,216)=30.1, p<$ $.001) .^{2}$ These data show that sets composed of complementary features were perceived to be, on average, more attractive than sets composed of noncomplementary features. Thus, in the absence of complementarity effects, respondents should have been more likely to postpone their decision when choosing from the less attractive sets composed from noncomplementary features-a prediction that is directionally opposite to hypothesis 1 .

Choice-Share Analyses. Hypothesis 1 predicted that choice deferral is a function of the complementarity of the features differentiating choice alternatives, such that choice deferral is greater for sets in which options are differentiated by complementary than by noncomplementary features. Each of the 73 respondents made four choice decisions yielding 289 observations in total (three missing data points). The data show that the respondents were more likely to postpone their choice when making a selection from a complementary assortment than they did when selecting from a noncomplementary assortment $(55.9 \%, N=145$, vs. $30.6 \%, N=144)$. This effect was directionally consistent for both product categories $(58.3 \%, N=72$, vs. $22.2 \%$, $N=72$ for the MP3 player, and $53.4 \%, N=73$, vs. $38.9 \%$, $N=72$ for the resort).

The significance of these data was tested using repeatedmeasures categorical data analysis (Stokes, Davis, and Koch 2001). The model represented the likelihood of delaying the choice as a function of feature type, product category, and their interaction. The data show a significant main effect of feature type $\left(\chi^{2}(1)=18.91, p<.001\right)$, indicating that complementary and noncomplementary features are likely to be associated with different choices. The (feature type) $\times$ (product category) interaction was significant as well $\left(\chi^{2}(1)=3.98, p<.05\right)$, indicating that the observed effect varied in strength for the two product categories. In particular, the effect was more pronounced in the MP3 category

\footnotetext{
${ }^{2}$ Additional analyses using the highest rating (rather than the average of all ratings) yielded similar results.
} 
$\left(\chi^{2}(1)=18.37, p<.001\right)$ and less pronounced for choices among different resorts $\left(\chi^{2}(1)=3.06, p<.10\right)$. These data are consistent with the experimental predictions advanced in hypothesis 1 .

The observed data pattern was associated with the fact that each complementary feature added a new dimension to a consumer's ideal point, which in turn lowered the attractiveness of the options lacking that particular feature. This prediction, however, is contingent on the dispersion of relative attractiveness of the complementary features. In particular, one can argue that the potential negative impact of complementary features on choice will be more pronounced for consumers who perceive these features to be equally important and less pronounced in cases when some of the features dominate the others. Indeed, the presence of a dominant feature is likely to be perceived as a reason for choice and to increase the likelihood of relying only on that most important attribute (Bettman, Luce, and Payne 1998; Tversky, Sattath, and Slovic 1988); as a result, the potential negative impact of adding complementary features will be less pronounced.

The above discussion implies that the proposition advanced in hypothesis 1 can be further tested by examining whether the impact of feature type on choice likelihood is also a function of the dispersion of the features' relative importance. For that purpose, individual decisions were assigned to one of the two attribute importance conditions based on the pattern of dispersion of the relative attractiveness of the product features. Thus, 56 decisions in which all features were perceived to be equally attractive (corresponding to a 2-2-2-2-2 distribution of the 10 points in the relative importance evaluation task) were classified into the equal importance condition, whereas the remaining 233 choices were classified into the dominant feature condition.

The data show that respondents who perceived complementary features to be equally attractive were more likely to postpone their decision than respondents who perceived some of the features to be more attractive than the others $(87.5 \%, N=16$, vs. $51.9 \%, N=129)$. In the case of noncomplementary features, the corresponding effects were in the same direction, although less pronounced $(32.5 \%$, $N=40$, vs. $29.8 \%, N=104)$. The significance of these differences was examined by testing a model in which the likelihood of delaying the choice was given as a function of the feature's relative importance, feature type, and their interaction. The data show a significant interaction between the relative importance of product features and feature type $\left(\chi^{2}(1)=3.99, p<.05\right)$, which suggests that the strength of the effect predicted in hypothesis 1 is also a function of the dispersion of the relative importance of the complementary and noncomplementary product features. The data further show that the moderating effect of feature importance was significant only in the case of complementary features; respondents who perceived features to be equally important were more likely to delay their choice $\left(\chi^{2}(1)=5.79, p<\right.$ $.05)$. This data pattern supports the propositions advanced in hypothesis 1 .

\section{Discussion}

The experimental data lend support to the notion that complementarity of the features differentiating choice options has a significant impact on choice. In particular, the data show that respondents were less likely to make a choice from sets in which options were differentiated by complementary features than from sets differentiated by noncomplementary features. These findings are consistent with the predictions made by hypothesis 1 .

The data further show that the impact of feature complementarity on choice is a function of the dispersion of the relative importance of these features. Thus, the choice likelihood was lower in cases when features were perceived to be equally important than in cases when some of the features were perceived to be more important than others. These findings are consistent with the finding that consumers without an articulated ideal point are likely to have weaker preferences for the selected alternative and are more likely to switch (Chernev 2003b; Hoeffler and Ariely 1999). Building on prior research, the data reported in experiment 1 further show that the ideal point availability effect is further moderated by the complementarity of the features differentiating choice alternatives. In particular, it is shown that the ideal point availability is more likely to strengthen consumer preferences for choices from noncomplementary than from complementary sets.

More generally, this research argues that adding options differentiated by complementary features highlights the deficiencies of the options in the original set, thus decreasing their overall attractiveness. This argument also implies that the decrease in the attractiveness of the options in the set should be proportional to the set size. Indeed, because each option differentiated by a complementary feature adds a new dimension on which all other options are likely to be perceived inferior, increasing the number of options differentiated by complementary features should also lead to an increase in the number of dimensions on which the majority of the options are inferior. As a result, as the number of complementary options increases, the relative attractiveness of each individual option will decrease. The decrease in the relative attractiveness of choice options is then likely to lead to a lower purchase probability.

The above discussion predicts that increasing assortment by adding options differentiated by complementary features inadvertently decreases the attractiveness of the options in the original set by highlighting their deficiencies on the attributes defined by the complementary features. It is further proposed that this effect will be more pronounced for options differentiated by complementary features than for options differentiated by noncomplementary features. This prediction can be more formally stated as follows:

H2: Feature complementarity moderates the impact of the size of the choice set on purchase likelihood. In particular, increasing the size of a given set by adding options differentiated by complementary features is more likely to have a negative impact 
on choice likelihood than adding options differentiated by noncomplementary features.

This hypothesis is tested in the following experiment.

\section{EXPERIMENT 2}

The goal of this experiment was to examine whether complementarity of features differentiating choice alternatives moderates the impact of set size on choice (hypothesis 2). This prediction is tested by comparing the likelihood of purchase from smaller (two options) versus larger (five options) sets, which are differentiated by either complementary or noncomplementary features. The basic idea was to extend a binary set by adding options differentiated by either complementary or noncomplementary features and, in this context, to compare the change in the purchase probability associated with the increase of the set size as a function of feature complementarity. The experimental methodology is presented in more detail in the following section.

\section{Method}

One hundred seventy-six Northwestern University undergraduates were recruited to participate in an experiment on consumer decision making. The stimuli included products from two categories: an MP3 player and toothpaste. Each option was described on four attributes such that the first attribute was composed of features unique to each option, with the other three attributes being features common to all options. Features used to describe the MP3 players were similar to the ones in the first experiment: options, color, weight, and warranty. The toothpaste was described on functionality, flavor, fluoride content, and packaging. A more detailed description of these features is given in appendix table A2.

Feature complementarity was manipulated by varying the type of features differentiating choice options, as follows. In the complementary condition, MP3 players varied in terms of options (extended battery life, memory expansion slot, extra sturdy construction, shock-resistant play, or userfriendly design) and had three common features: color, weight, and warranty. The weight and the warranty were the same in all conditions ( $14 \mathrm{oz}$. and 12 mo., respectively), and the color was rotated (silver, white, black, blue, or red) so that each respondent was given only one color, but the particular color varied across respondents. In contrast, in the noncomplementary condition, the MP3 players varied in color (silver, white, black, blue, or red) and had three common features: options, weight, and warranty. The weight and the warranty were the same as in the complementary condition (14 oz. and 12 mo., respectively), and options were rotated across conditions (extended battery life, memory expansion slot, extra sturdy construction, shock-resistant play, or user-friendly design). The rotation of different options and colors as common features was done to counterbalance any potential attribute-specific effects.

The toothpaste stimuli were organized in a similar manner.
Thus, toothpastes in the complementary condition varied on their primary function (cavity prevention, tartar prevention, fights gingivitis, advanced cleaning, or teeth sensitivity) and had three common features: flavor, fluoride content, and packaging. The fluoride content and packaging were identical across all conditions, whereas the flavor was rotated (peppermint, mint, citrus, strawberry, or cinnamon) so that each respondent was given only one flavor, but the flavor type varied across respondents. In contrast, in the noncomplementary condition, the toothpastes varied in flavor (peppermint, mint, citrus, strawberry, or cinnamon) and had three common features: primary function, fluoride content, and packaging. The fluoride and packaging were the same as in the complementary condition, and the primary function was rotated across conditions (cavity prevention, tartar prevention, fights gingivitis, advanced cleaning, or teeth sensitivity).

Respondents were given choice sets composed of either two or five alternatives. The larger set included all five options shown in appendix table A2, whereas the smaller set consisted of a subset of two randomly selected options. Respondents were asked to make a choice from each of the two product categories and were given the option of not choosing either of the available alternatives. Respondents were also asked to provide the rationale for their decisions.

The overall experimental design was a 2 (feature complementarity: high vs. low) $\times 2$ (choice set: large vs. small) $\times 2$ (product category: MP3 player vs. toothpaste) factorial design in which feature complementarity and the size of the choice set were manipulated between subjects. The experimental design also included several counterbalancing procedures, such as using different subsets of complementarynoncomplementary features in the binary sets to control for any potential feature-specific effects. The experiment was conducted online and respondents were recruited via e-mail.

\section{Results}

Manipulation Check. The experimental stimuli were pretested to ensure that respondents indeed perceived product features to be complementary. Because the MP3 player was pretested in experiment 1 , this manipulation check examined only the toothpaste stimuli. The procedure and analyses were identical to the ones used in the first experiment in which respondents had to create their ideal product. The data show that the total number of features used to describe the respondents' ideal point varied across the two feature types and that the mean for the complementary features was significantly higher than the mean for the noncomplementary features $(M=3.63$ vs. $M=1.16 ; F(1,22)=93.6$, $p<.001)$. These data are consistent with the goals of the manipulation procedure.

Choice-Share Analyses. Hypothesis 2 argued that the impact of assortment on probability of purchase is moderated by the complementarity of the alternatives and that noncomplementary assortments are associated with a greater probability of purchase than complementary assortments. Each of the 176 respondents made two choices, one per 
product category, yielding 352 observations in total. The data indicate that respondents were more likely to delay their choice when making a selection from a complementary assortment than when choosing from a noncomplementary assortment. In particular, 57.4\% of the respondents opted to postpone their choice when selecting among options differentiated by complementary features, compared with only $34.7 \%$ of respondents who had to select among noncomplementary options. More important, this effect was a function of the size of the choice set. Thus, when choosing among options differentiated by complementary features, $65.1 \%$ of the respondents decided to postpone their choice when choosing from a larger set, compared with $50 \%$ of those choosing from a smaller set. For sets differentiated by noncomplementary features the effect was in the opposite direction: $26.7 \%$ of the respondents decided to postpone their choice when choosing from a larger set compared with $42.2 \%$ of those choosing from a smaller set. This effect was directionally consistent for both product categories as shown in table 1, panel A.

The significance of these data was tested using a model that represented choice deferral as a function of feature type, set size, product category, and their interactions. The data show that the main effect of feature complementarity on choice deferral was significant $\left(\chi^{2}(1)=19.73, p<.001\right)$. More important, the (feature type) $\times$ (set size) interaction was significant $\left(\chi^{2}(1)=9.35, p<.005\right)$, which indicates that the observed complementarity effect varied in strength as a function of the size of the choice set. In particular, for sets differentiated by complementary features, the impact of increasing the set size was significant $\left(\chi^{2}(1)=4.27, p<\right.$ $.05)$, as was the impact of increasing the set size for options differentiated by noncomplementary features $\left(\chi^{2}(1)=\right.$ $5.08, p<.05)$. These data lend support to hypothesis 2 .

The observed effect was also consistent across the two product categories as indicated by the nonsignificant (feature type $) \times($ set size $) \times$ (product category) interaction $\left(\chi^{2}(1)<\right.$ $1)$. The main category effect was significant $\left(\chi^{2}(1)=\right.$ $10.17, p<.001)$, indicating that respondents were more likely to postpone their decision when buying an MP3 player than when buying a toothpaste. More important, the moderating effect of feature complementarity on choices from large versus small sets was significant for each of the two product categories $\left(\chi^{2}(1)=4.37, p<.05\right.$ for the MP3 player, and $\chi^{2}(1)=4.98, p<.05$ for the toothpaste). These data are consistent with hypothesis 2 .

Choice-Justification Analyses. Respondents' selfreported choice reasons offer further insight into the decision processes underlying their choices. Each of the 176 respondents was asked to provide reasons for their choices in each of the two product categories, yielding 331 observations in total (21 missing data points). The methodology of coding individuals' reasons was similar to the one used for coding thought protocols (Ericsson and Simon 1980; Wright 1974).

Individuals' responses were blind coded by two independent judges into one of the following categories: (1) missingideal reasons, which referred to the absence of an option
TABLE 1

CHOICE DEFFERAL AND REASONING AS A FUNCTION OF FEATURE COMPLEMENTARITY AND SET SIZE (EXPERIMENT 2)

\begin{tabular}{|c|c|c|}
\hline \multirow[b]{2}{*}{ Stimuli and feature type } & \multicolumn{2}{|c|}{ Set size } \\
\hline & $\begin{array}{l}\text { Large (\%) } \\
\text { (5 options) }\end{array}$ & $\begin{array}{c}\text { Small (\%) } \\
\text { (2 options) }\end{array}$ \\
\hline \multicolumn{3}{|c|}{$\begin{array}{l}\text { A. Choice deferral as a function } \\
\text { of feature complementar- } \\
\text { ity and set size: }\end{array}$} \\
\hline \multicolumn{3}{|c|}{ MP3 player: } \\
\hline Complementary & $74.4(43)$ & $55.6(45)$ \\
\hline Noncomplementary & $37.2(43)$ & $48.9(45)$ \\
\hline \multicolumn{3}{|l|}{ Toothpaste: } \\
\hline Complementary & $55.8(43)$ & $44.4(45)$ \\
\hline Noncomplementary & $16.3(43)$ & $35.6(45)$ \\
\hline \multicolumn{3}{|l|}{ Total: } \\
\hline Complementary & $65.1(86)$ & $50.0(90)$ \\
\hline Noncomplementary & $26.7(86)$ & $42.2(90)$ \\
\hline \multicolumn{3}{|c|}{$\begin{array}{l}\text { B. Complementary (missing- } \\
\text { ideal) reasoning as a } \\
\text { function of feature com- } \\
\text { plementarity and set size: }\end{array}$} \\
\hline \multicolumn{3}{|c|}{ MP3 player: } \\
\hline Complementary & $47.5(40)$ & $14.6(41)$ \\
\hline Noncomplementary & $2.5(40)$ & $4.5(44)$ \\
\hline \multicolumn{3}{|l|}{ Toothpaste: } \\
\hline Complementary & $35.0(40)$ & $12.2(41)$ \\
\hline Noncomplementary & $2.4(41)$ & $4.5(44)$ \\
\hline \multicolumn{3}{|l|}{ Total: } \\
\hline Complementary & $41.3(80)$ & $13.4(84)$ \\
\hline Noncomplementary & $2.5(81)$ & $4.5(88)$ \\
\hline
\end{tabular}

NotE.-Cell sizes are given in parentheses.

integrating features offered by different options in the set (e.g., "I hope there is one model that has all of the good features"); (2) feature-specific reasons, which referred to an individual's preference for a particular feature (e.g., "I like mint flavor"); (3) set-size reasons, which referred to a general preference for a larger set (e.g., "I would like more options to choose from"); and (4) miscellaneous reasons that could not be classified in either of the first three categories. Overall, 50 of the responses were classified into the missingideal category, 128 were classified as feature-specific, 55 were set-size specific, and the remaining 98 were classified as miscellaneous.

This research argued that differentiation by complementary features highlights the fact that other available options are likely to have deficiencies on the attributes defined by the complementary features. This argument implies that the ideal point created by aggregating specific features was more likely to be used as a reference point for choices from complementary than from noncomplementary assortments. This prediction was empirically tested by comparing the frequencies of respondents' use of the missing-ideal rationale across the experimental conditions.

Quantitative analysis shows that respondents were more likely to use the missing-ideal rationale in cases when choosing from complementary than when choosing from noncomplementary sets. Thus, $27.2 \%$ of respondents used the 
missing-ideal rationale when selecting among options differentiated by complementary features, compared with only $3.6 \%$ of those who had to select among noncomplementary options. More important, this effect was also a function of the size of the choice set. For choices among options differentiated by complementary features, $41.3 \%$ of the respondents used the missing-ideal rationale when choosing from larger sets compared with $13.4 \%$ of those choosing from smaller sets. For sets differentiated by noncomplementary features, there was essentially no effect: $2.5 \%$ versus $4.5 \%$. This data pattern was consistent for both product categories, as shown in table 1, panel B.

The significance of this data pattern was tested using a model that represented respondents' reliance on the missingideal rationale as a function of feature type, set size, product category, and their interactions. Categorical analysis shows a significant (feature type) $\times$ (set size) interaction $\left(\chi^{2}(1)=4.91, p<.05\right)$, which suggests that respondents' reliance on the missing-ideal rationale was a function of both feature complementarity and the size of the decision set. In particular, for options differentiated by complementary features, larger sets were associated with greater reliance on the missing-ideal rationale than smaller sets $\left(\chi^{2}(1)=14.39, p<.001\right)$. In contrast, for options differentiated by noncomplementary features, the corresponding effect was nonsignificant $\left(\chi^{2}(1)<1\right)$. This data pattern was consistent across the two product categories, as indicated by the nonsignificant (feature type) $\times($ set size $) \times$ (product category) interaction $\left(\chi^{2}(1)<1\right)$. These findings are consistent with the theory that differentiating by complementary features highlights the deficiencies of the other options, which, in turn, lowers the attractiveness of choice alternatives and increases the likelihood of choice deferral.

\section{Discussion}

The data reported in this experiment are consistent with the proposition that feature complementarity moderates the impact of the size of the choice set on probability of purchase. The data show that when choosing from sets differentiated by complementary features, respondents were more likely to defer their choice in the context of a larger than a smaller assortment. In contrast, when choosing from sets differentiated by noncomplementary features, the opposite was true: respondents were more likely to defer their choice in the context of a smaller than a larger assortment. Analysis of respondents' self-explicated reasons suggests that the greater probability of deferral for choices made from complementary assortments was associated with the absence of the ideal option, which combines the complementary features offered by different options. These findings are consistent with the theory advanced in this research and with the predictions made by hypothesis 2 .

The discussion so far has focused on a scenario in which all choice alternatives belong to the same category and are not divided into subsets (e.g., by brand). An alternative strategy to test the research propositions is to examine a scenario in which options are partitioned in such a manner that con- sumers can choose between a single nondifferentiated option and a subset of either complementary or noncomplementary options. To illustrate, consider a set composed of two brands in which brand $\mathrm{A}$ is represented by a single option and brand $\mathrm{B}$ is represented by several either complementary or noncomplementary options.

Conceptually, this scenario represents constrained choice (Kahn, Moore, and Glazer 1987), in which the decision set is partitioned by brand, and consumers have to choose between a single nondifferentiated option identified by one of the brands and a subset of options identified by the other brand. Building on the prior discussion, it is argued that in the above scenario, the choice share of the subset of options will be greater when options in this subset are differentiated by noncomplementary rather than by complementary features. The prediction is that consumers will be more likely to choose from a brand-specific subset differentiated by noncomplementary features than from a subset differentiated by complementary features. More formally, this argument can be summarized as follows:

H3: The choice share of a given subset of options is a function of the complementarity of its alternatives. In particular, subsets differentiated by noncomplementary features are associated with a greater probability of purchase than subsets differentiated by complementary features.

This hypothesis is tested in the following experiment.

\section{EXPERIMENT 3}

\section{Method}

One hundred and ten Northwestern University undergraduates were recruited to participate in the experiment. They were informed that the choice task would involve making several hypothetical product choices in different product categories. Respondents were paid \$5 for participating.

The experimental stimuli consisted of 20 choice sets that varied in complementarity, set composition, and product category. Each alternative was described on four attributes: one attribute composed of either complementary or noncomplementary features and the other three composed of common features (appendix table A3). Choice alternatives were organized in sets of either two or five options. Options were grouped by brand so that one of the brands (brand A) had a single offering and the other brand (brand B) had four different offerings. As a result, there were four binary sets in which brand $\mathrm{A}$ was paired with different versions of brand $\mathrm{B}$, and one set that included all five alternatives. Schematically, these choice sets can be represented as $\left(A, B_{1}\right),(A$, $\left.B_{2}\right),\left(A, B_{3}\right),\left(A, B_{4}\right)$, and $\left(A, B_{1}, B_{2}, B_{3}, B_{4}\right)$, where $B_{1}-B_{4}$ are either complementary or noncomplementary variants of brand B. The product categories used in this experiment were shaving cream and toothpaste. The overall experimental design was a 2 (feature complementarity: high vs. low) $\times 5$ (choice set: binary vs. all-inclusive) $\times 2$ (product 
category: shaving cream vs. toothpaste) within-subjects design.

Respondents were first asked to make four binary choices from sets in which brand A was paired with each of the four versions of brand B. Next, respondents were asked to choose from a set containing five alternatives: brand $\mathrm{A}$ and the four versions of brand B. Overall, each respondent made five decisions in each of the two product categories and for each of the two feature types. At the end of the experiment, respondents were also asked to write down their reasons for choosing from the all-inclusive set.

The experimental design was based on the rationale that the impact of feature complementarity can be captured by comparing the pattern of respondents' choices displayed in the binary sets and their choices from the all-inclusive set. Because feature complementarity is defined relative to the other alternatives, its impact should be less pronounced in the context of the binary than in the the all-inclusive sets. In this context, one approach to measure the impact of feature complementarity is to examine the consistency of respondents' preferences across the binary and the all-inclusive sets. According to the principle of independence of irrelevant alternatives, an individual who prefers brand $\mathrm{B}$ to brand $\mathrm{A}$ in any of the binary sets should then also choose brand $\mathrm{B}$ in the all-inclusive set. If, however, feature complementarity lowers the relative attractiveness of the alternatives in the all-inclusive set, as predicted in this research, then choices from complementary sets are more likely to lead to preference reversals, so that individuals who prefer brand $B$ to brand $\mathrm{A}$ in some or all of the binary sets nevertheless choose brand $\mathrm{A}$ from the all-inclusive set.

\section{Results}

Manipulation Check. The experimental stimuli were pretested to ensure that product features were indeed perceived to be complementary by the respondents. Because toothpaste was pretested in experiment 2 , this manipulation check examined only the shaving cream category. The procedure and analyses were identical to the ones used in the first two experiments. The data show that the total number of features used to describe the respondents' ideal point varied across the two feature types, so that the mean for the complementary features was significantly higher than the mean for the noncomplementary features $(M=2.92$ vs. $M=1.09 ; F(1,22)=29.1, p<.001)$. This finding is consistent with the goals of the experimental manipulation.

Choice-Share Analyses. Hypothesis 3 predicted that the choice share of a given subset of options is moderated by the complementarity of the alternatives, so that subsets differentiated by noncomplementary features are associated with a greater probability of purchase than subsets differentiated by complementary features. This hypothesis was tested by comparing the pattern of responses across the experimental conditions. For each of the two categories and assortment types, respondents were asked to make choices from five sets: four binary sets that paired option $\mathrm{A}$ with one of the four different variants of option B and the fifth set that offered a choice between option $A$ and the four variants of option B. Based on the pattern of choices from these sets, a combined measure of preference consistency was calculated as follows.

Respondents were considered to have consistent preferences in one of the two scenarios: (1) respondents selected option $\mathrm{A}$ in each of the four binary choices and then also selected option $\mathrm{A}$ from the all-inclusive set $(\mathrm{A} \rightarrow \mathrm{A})$, and (2) respondents selected option $\mathrm{B}$ in each of the four binary choices and then also selected option $\mathrm{B}$ from the all-inclusive set $(\mathrm{B} \rightarrow \mathrm{B})$. If, however, respondents selected option $\mathrm{A}$ in all binary sets and then selected option $B$ from the all-inclusive set $(A \rightarrow B)$, or they selected option $B$ in some of the binary sets and then selected option $A$ in the extended set $(\mathrm{B} \rightarrow \mathrm{A})$, their preferences were considered to be inconsistent. To illustrate, if a respondent prefers $A$ to $B_{1}, B_{2}$, and $B_{3}$ but prefers $\mathrm{B}_{4}$ to $\mathrm{A}$, then choosing option $\mathrm{A}$ from a set comprising $\mathrm{B}_{1}-\mathrm{B}_{4}$ is considered an indication of preference inconsistency. Hypothesis 3 implies that such preference inconsistency is more likely to occur in the context of complementary than noncomplementary assortments.

Consistent with the above discussion, the choices made by each respondent were classified into one of the following categories: (1) $\mathrm{A} \rightarrow \mathrm{A}$, in which brand $\mathrm{A}$ was preferred in both binary and the all-inclusive sets; (2) B $\rightarrow \mathrm{B}$, in which brand $\mathrm{B}$ was preferred in both binary and all-inclusive sets; (3) $\mathrm{A} \rightarrow \mathrm{B}$, in which respondents selected option $\mathrm{A}$ in the binary sets but preferred option $\mathrm{B}$ in the all-inclusive set; and (4) B $\rightarrow$ A, in which brand B was preferred in at least one binary set, but brand A was chosen from the all-inclusive set. Each of the 110 respondents made choices from either complementary or noncomplementary assortments in two product categories, yielding 437 observations in total (three missing data points).

The data summarized in table 2 show that whereas the majority of the respondents $(88.1 \%)$ displayed consistent preferences across the binary and the all-inclusive sets, a substantial number of responses showed inconsistencies.

TABLE 2

PREFERENCE CONSISTENCY AS A FUNCTION OF PRODUCT ASSORTMENT AND FEATURE COMPLEMENTARITY (EXPERIMENT 3)

\begin{tabular}{|c|c|c|c|c|}
\hline \multirow[b]{2}{*}{ Stimuli and feature type } & \multicolumn{4}{|c|}{ Choice pattern (\%) } \\
\hline & $\overline{A \rightarrow A}$ & $\mathrm{~B} \rightarrow \mathrm{B}$ & $A \rightarrow B$ & $\overline{B \rightarrow A}$ \\
\hline \multicolumn{5}{|l|}{ Shaving cream: } \\
\hline Complementary $(N=110)$ & 19.1 & 60.9 & 1.8 & 18.2 \\
\hline Noncomplementary $(N=109)$ & 25.7 & 67.9 & 3.7 & 2.8 \\
\hline \multicolumn{5}{|l|}{ Toothpaste: } \\
\hline Complementary $(N=110)$ & 14.5 & 70.9 & .9 & 13.6 \\
\hline Noncomplementary $(N=108)$ & 40.7 & 52.8 & 1.9 & 4.6 \\
\hline
\end{tabular}


More important, these preference inconsistencies were asymmetric; they were more pronounced for complementary features and in a direction favoring brand $\mathrm{A}$ in the allinclusive set. Thus, in the case of complementary assortments, $15.9 \%$ of respondents selected brand $\mathrm{A}$ in the allinclusive set after choosing brand $\mathrm{B}$ in at least one of the binary sets, compared with only $3.7 \%$ of respondents in the case of noncomplementary assortments. Furthermore, for choices from complementary assortments, this preference reversal was more pronounced when favoring brand $\mathrm{A}$ than any of the options in the product line represented by brand B: only $1.4 \%$ of respondents preferred brand B from the all-inclusive set after selecting brand $\mathrm{A}$ in all binary sets $(\mathrm{A} \rightarrow \mathrm{B})$, compared with $15.9 \%$ of respondents who displayed a preference reversal in the opposite direction $(\mathrm{B} \rightarrow \mathrm{A})$. This pattern of reversals was consistent across the two product categories.

The significance of these data was tested using repeatedmeasures categorical data analysis. Several different models were tested in which preference reversals were given as a function of feature type, product category, and their interaction. In particular, the test examined whether preference reversals in either direction $(\mathrm{A} \rightarrow \mathrm{B}$ and $\mathrm{B} \rightarrow \mathrm{A}$ vs. $\mathrm{A} \rightarrow \mathrm{A}$ and $\mathrm{B} \rightarrow \mathrm{B}$ ) varied significantly as a function of feature type and product category. The data show that the likelihood of preference reversals was a function of feature type $\left(\chi^{2}(1)=11.34, p<.001\right)$, whereas the effect of product category was nonsignificant $\left(\chi^{2}(1)<1\right)$.

An additional test involved examining the significance of the asymmetric pattern of preference reversals $(\mathrm{A} \rightarrow \mathrm{B}$ vs. $\mathrm{B} \rightarrow \mathrm{A})$ as a function of feature type and product category. The data show that respondents were significantly more likely to choose the single brand A from the all-inclusive set when the product line offered by brand B was differentiated by complementary features $\left(\chi^{2}(1)=6.62, p=\right.$ $.01)$. This effect was consistent across the two product categories, as indicated by the nonsignificant (feature type) $\times$ (product category) interaction $\left(\chi^{2}(1)<1\right)$. These data support the predictions outlined in hypothesis 3 .

\section{Discussion}

The data reported in this experiment are consistent with the proposition that the choice share of a given subset of options is a function of the complementarity of the features differentiating these options. This proposition was tested by comparing the consistency of respondents' preferences when choosing between two brands: one featuring a single option and the other featuring either a complementary or noncomplementary product line. The data show that noncomplementary subsets tend to have a greater choice share than complementary subsets.

Findings reported in this experiment are consistent with prior research on the role of comparisons and grouping in choice, which demonstrates that when the options being compared have both advantages and disadvantages, comparisons between options make each option less attractive (Brenner, Rottenstreich, and Sood 1999; see also Glazer,
Kahn, and Moore 1991; Tversky and Sattath 1979). The data reported in this experiment lend further support for these findings, demonstrating that the effect of grouping on choice is also moderated by the complementarity of the features differentiating choice options, whereby the lone alternative effect tends to be more pronounced for complementary than for noncomplementary features.

\section{GENERAL DISCUSSION}

This research demonstrates, for the first time, that the probability of purchase from a given assortment is contingent on the complementarity of the features differentiating its options. In particular, noncomplementary choice sets were shown to be associated with a greater probability of purchase compared with complementary sets. This effect was attributed to the fact that adding options differentiated by complementary features to a given choice set tends to decrease the overall attractiveness of the options in the set by highlighting the deficiencies of the options on the attribute defined by that feature.

This proposition was supported by the data from three empirical studies. The first experiment examined the impact of feature complementarity on purchase likelihood. This experiment shows that sets in which options are differentiated by noncomplementary features are associated with a greater probability of purchase than sets in which options are differentiated by complementary features. The second experiment examined how feature complementarity moderates the impact of assortment on choice. This experiment demonstrates that increasing the size of a given set by adding options differentiated by noncomplementary features is less likely to have a negative impact on choice likelihood than adding options differentiated by complementary features. Finally, the third experiment examined a scenario in which options were partitioned such that consumers could choose between a single nondifferentiated option and either a complementary or noncomplementary assortment. The data reported in this experiment show that the choice share of a subset of options is greater when the options are differentiated by noncomplementary rather than by complementary features.

The research presented in this article further contributes to the extant marketing and decision literature by demonstrating that feature complementarity moderates the impact of assortment on choice. Thus, recent research has shown that increasing the size of the choice set may lower the overall probability of purchase (Chernev 2003a; Dhar 1997; Huffman and Kahn 1998; Iyengar and Lepper 2000). Most of this research, however, has examined the impact of assortment on choice without explicitly focusing on the potential relationship between features describing choice alternatives (although see Hoch, Bradlow, and Wansink 1999; Kahn and Wansink 2004). As a result, it has been implicitly assumed that all product features influence consumer decision processes and choice in a similar manner. In fact, most prior studies have examined a scenario in which alternatives are differentiated primarily on noncomplementary 
features, such as size, color, shape, and flavor. Building on prior research, this article demonstrates that the impact of assortment on choice is further moderated by the complementarity of the features differentiating choice alternatives and that the adverse impact of assortment on choice is likely to be more pronounced in assortments differentiated by complementary features.

This research argued that extending a product line by differentiation on complementary features leads to a lower choice probability than does extending a product line by differentiation on noncomplementary features. This argument was based on the notion that adding new features increases the number of relevant dimensions on which products are evaluated and that the lack of a given feature is then interpreted as a potential loss, which lowers the overall attractiveness of the choice alternatives. It is not clear, how- ever, how consumers make inferences about an option's performance on an attribute defined by a feature differentiating another option. Thus, one could argue that an option's superiority on a given attribute leads to discounting its performance on the other attributes by way of compensatory inferences, such that a superior value on one attribute implies inferiority on other attributes (Chernev 2004; Chernev and Carpenter 2001). To illustrate, toothpaste that emphasizes cavity prevention might be perceived to have below-average tartar protection. Thus, the addition of an equally priced option that is superior on a given attribute might invoke compensatory inferences that will further hurt the other options' ratings on that attribute. Investigating the role of compensatory inferences in moderating the impact of complementary and noncomplementary features on choice is a promising venue for further research.

\section{APPENDIX}

TABLE A1

AN OVERVIEW OF THE STIMULI DESIGN IN EXPERIMENT 1

\begin{tabular}{|c|c|c|c|c|c|}
\hline Product features & Option 1 & Option 2 & Option 3 & Option 4 & Option 5 \\
\hline \multicolumn{6}{|l|}{ MP3 player: } \\
\hline $\begin{array}{l}\text { Complementary features } \\
\text { Noncomplementary }\end{array}$ & User-friendly design & Extended battery life & Clear sound & Extra sturdy & Shock-resistant play \\
\hline features & Silver & White & Black & Blue & Red \\
\hline \multicolumn{6}{|l|}{ Resort: } \\
\hline Complementary features & Fantastic beaches & $\begin{array}{l}\text { Convenient transpor- } \\
\text { tation }\end{array}$ & Vibrant nightlife & Excellent restaurants & Exceptional service \\
\hline $\begin{array}{l}\text { Noncomplementary } \\
\text { features }\end{array}$ & Bermuda & Bahamas & Barbados & Antigua & Aruba \\
\hline
\end{tabular}

TABLE A2

AN OVERVIEW OF THE STIMULI DESIGN IN EXPERIMENT 2

\begin{tabular}{|c|c|c|c|c|c|}
\hline Product features & Option 1 & Option 2 & Option 3 & Option 4 & Option 5 \\
\hline \multicolumn{6}{|l|}{ MP3 player: } \\
\hline Complementary features (options) ${ }^{a}$ & $\begin{array}{l}\text { Extended battery } \\
\text { life }\end{array}$ & $\begin{array}{l}\text { Memory expan- } \\
\text { sion slot }\end{array}$ & Extra sturdy & $\begin{array}{l}\text { Shock-resistant } \\
\text { play }\end{array}$ & $\begin{array}{l}\text { User-friendly } \\
\text { design }\end{array}$ \\
\hline Noncomplementary features (color) ${ }^{\mathrm{b}}$ & Silver & White & Black & Blue & Red \\
\hline \multicolumn{6}{|l|}{ Toothpaste: } \\
\hline Complementary features (functionality) ${ }^{c}$ & Cavity prevention & Tartar prevention & Fights gingivitis & Advanced cleaning & Sensitive teeth \\
\hline Noncomplementary features (flavor) ${ }^{d}$ & Peppermint & Mint & Citrus & Strawberry & Cinnamon \\
\hline \multicolumn{6}{|c|}{ 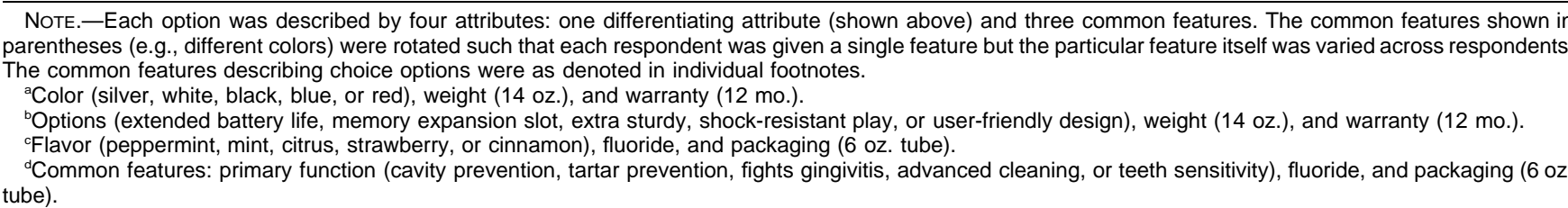 } \\
\hline
\end{tabular}


TABLE A3

AN OVERVIEW OF THE STIMULI DESIGN USED IN EXPERIMENT 3

\begin{tabular}{|c|c|c|c|c|c|}
\hline \multirow[b]{2}{*}{ Product features } & \multirow{2}{*}{$\frac{\text { Brand A }}{\text { Option } 1}$} & \multicolumn{4}{|c|}{ Brand B } \\
\hline & & Option 1 & Option 2 & Option 3 & Option 4 \\
\hline \multicolumn{6}{|l|}{ Shaving cream: } \\
\hline Complementary features & Complete formula & $\begin{array}{l}\text { Hydrates and mois- } \\
\text { turizes }\end{array}$ & Extra skin protection & $\begin{array}{l}\text { Revitalizes and } \\
\text { reconditions }\end{array}$ & $\begin{array}{l}\text { Penetrates dirt and } \\
\text { oil }\end{array}$ \\
\hline $\begin{array}{l}\text { Noncomplementary } \\
\text { features }\end{array}$ & $\begin{array}{l}\text { Cool wave: clean } \\
\text { and crisp }\end{array}$ & $\begin{array}{l}\text { Wild rain: invigorating } \\
\text { and fresh }\end{array}$ & $\begin{array}{l}\text { Pacific light: fresh } \\
\text { and light }\end{array}$ & $\begin{array}{l}\text { Breeze: reviving and } \\
\text { oriental }\end{array}$ & $\begin{array}{l}\text { Arctic peak: bold and } \\
\text { exhilarating }\end{array}$ \\
\hline $\begin{array}{l}\text { Toothpaste: } \\
\text { Complementary features }\end{array}$ & Triple action & Cavity prevention & Tartar protection & Rejuvenating & Advanced cleaning \\
\hline features & Mint & Herbal & Cinnamon & Citrus & Strawberry \\
\hline
\end{tabular}

NOTE.-Each option was described by four attributes: one differentiating attribute (shown above) and three common features. The common features describing the shaving cream were vitamin E, aloe, and glycerin; the common features describing toothpaste were baking soda, fluoride, and packaging. Shaving cream brand names were identified as Nivea and Gillette; toothpastes were identified as Pepsodent and Mentadent.

[Dawn Iacobucci served as editor and Stephen Nowlis served as associate editor for this article.]

\section{REFERENCES}

Bettman, James R., Mary Frances Luce, and John W. Payne (1998), "Constructive Consumer Choice Processes," Journal of Consumer Research, 25 (December), 187-217.

Brenner, Lyle, Yuval Rottenstreich, and Sanjay Sood (1999), "Comparison, Grouping, and Preference," Psychological Science, 10 (May), 225-29.

Chernev, Alexander (2003a), "Product Assortment and Individual Decision Processes," Journal of Personality and Social Psychology, 85 (July), 151-62.

- (2003b), "When More Is Less and Less Is More: The Role of Ideal Point Availability and Assortment in Consumer Choice," Journal of Consumer Research, 30 (September), 170-83.

- (2004), "Jack of All Trades or Master of One? Product Differentiation and Compensatory Reasoning in Consumer Choice," working paper, Kellogg School of Management, Northwestern University, Evanston, IL 60208.

Chernev, Alexander and Gregory S. Carpenter (2001), "The Role of Market Efficiency Intuitions in Consumer Choice: A Case of Compensatory Inferences," Journal of Marketing Research, 38 (August), 349-61.

Dhar, Ravi (1997), "Consumer Preference for a No-Choice Option," Journal of Consumer Research, 24 (September), 215-31.

Ericsson, Karl A. and Herbert A. Simon (1980), "Verbal Reports as Data," Psychological Review, 87 (July), 215-51.

Gentner, Dedre (1983), "Structure-Mapping: A Theoretical Framework for Analogy," Cognitive Science, 7 (April-June), 155-70.

Gentner, Dedre and Arthur B. Markman (1994), "Structural Alignment in Comparison: No Difference without Similarity," Psychological Science, 5 (May), 152-58.

Glazer, Rashi, Barbara E. Kahn, and William L. Moore (1991), "The Influence of External Constraints on Brand Choice: The Lone-Alternative Effect," Journal of Consumer Research, 18 (June), 119-27.

Henderson, James Mitchell and Richard E. Quandt (1958), Microeconomic Theory: A Mathematical Approach, New York: McGraw-Hill.

Hoch, Stephen J., Eric T. Bradlow, and Brian Wansink (1999), "The Variety of an Assortment," Marketing Science, 18 (4), 527-46.
Hoeffler, Steve and Dan Ariely (1999), "Constructing Stable Preferences: A Look into Dimensions of Experience and Their Impact on Preference Stability," Journal of Consumer Psychology, 8 (2), 113-39.

Huffman, Cynthia and Barbara E. Kahn (1998), "Variety for Sale: Mass Customization or Mass Confusion?" Journal of Retailing, 74 (4), 491-513.

Iyengar, Sheena S. and Mark R. Lepper (2000), "When Choice Is Demotivating: Can One Desire Too Much of a Good Thing?" Journal of Personality and Social Psychology, 79 (6), 995-1006.

Kahn, Barbara E., William L. Moore, and Rashi Glazer (1987), "Experiments in Constrained Choice," Journal of Consumer Research, 14 (June), 96-113.

Kahn, Barbara E. and Brian Wansink (2004), "The Influence of Assortment Structure on Perceived Variety and Consumption Quantities," Journal of Consumer Research, 30 (4), 519-33.

Lattin, James M. and Leigh McAlister (1985), "Using a VarietySeeking Model to Identify Substitute and Complementary Relationships among Competing Products," Journal of Marketing Research, 22 (August), 330-39.

Markman, Arthur B. and Dedre Gentner (1993), "Splitting the Differences: A Structural Alignment View of Similarity," Journal of Memory and Language, 32 (August), 517-35.

Markman, Arthur B. and Douglas L. Medin (1995), "Similarity and Alignment in Choice," Organizational Behavior and $\mathrm{Hu}$ man Decision Processes, 63 (August), 117-30.

Pindyck, Robert S. and Daniel L. Rubinfeld (2001), Microeconomics, 5th ed., London: Prentice-Hall.

Shafir, Eldar (1993), "Choosing versus Rejecting: Why Some Options Are Both Better and Worse than Others," Memory and Cognition, 21 (July), 546-56.

Stokes, Maura Ellen, Charles S. Davis, and Gary G. Koch (2001), Categorical Data Analysis Using the SAS System, 2nd ed., New York: Wiley.

Tversky, Amos and Shmuel Sattath (1979), "Preference Trees," Psychological Review, 86 (6), 542-73.

Tversky, Amos, Shmuel Sattath, and Paul Slovic (1988), "Contingent Weighting in Judgment and Choice," Psychological Review, 95 (July), 371-84.

Walters, R. G. (1991), "Assessing the Impact of Retail Price Promotions on Product Substitution, Complementary Purchase," Journal of Marketing, 55 (April), 17-28.

Wright, Peter (1974), "Analyzing Media Effects on Advertising Responses,” Public Opinion Quarterly, 38 (2), 192-205. 